\title{
Nefrologia Interventistica: una disciplina in evoluzione. L'esperienza di due centri italiani a confronto
}

\author{
Carlo Jovane ${ }^{1}$, Sara Dominijanni ${ }^{2}$, Emidio Costantini ${ }^{3}$, Barbara Campanati ${ }^{3}$, Davide Logaldo ${ }^{3}$, Alessia Centi ${ }^{2}$, \\ Francesco Londrino ${ }^{2}$, Paola Tatangelo ${ }^{2}$, Monica Bevilacqua ${ }^{2}$, Roberto Palumbo ${ }^{2}$, Alessandro Castiglioni ${ }^{1}$
}

${ }^{1}$ UOC Nefrologia e Dialisi, P.O. Gallarate, ASST Valle-Olona, Varese - Italia

${ }^{2}$ UOC Nefrologia e Dialisi, Ospedale Sant'Eugenio, ASL Roma 2, Roma - Italia

${ }^{3}$ UOC Chirurgia Vascolare, P.O. Busto Arsizio, ASST Valle-Olona, Varese - Italia

\begin{abstract}
Interventional Nephrology: an evolving discipline. The experience of two Italian centers
We describe the multidisciplinary work of nephrological realities belonging to two different Regional Health Systems, Lombardia and Lazio. The interventional nephrologist is a specialist in nephrology with ultra-specialist know-how for vascular access for dialysis. He is the coordinator of a team of vascular accesses and applies a team work with the other interventional nephrologists, with the vascular surgeon and the interventional radiologist, with whom he decides the diagnostic-therapeutic procedure to perform the best possible vascular access for that individual patient.
\end{abstract}

Keywords: A-V, Dialysis, FAV, Hub\&Spoke, Vascular Access Team

\section{Introduzione}

La Nefrologia Interventistica, una disciplina in continua crescita, è parte integrante della nefrologia. Nel passato, le procedure interventistiche erano spesso delegate ad altri specialisti, senza un continuum diagnostico con il nefrologo. In realtà, nel corso degli anni, il nefrologo è diventato abile a eseguire queste procedure in sicurezza, con successo e ottimi risultati (1). Al fine di migliorare sempre di più il percorso clinico di ogni singolo paziente con malattia renale cronica (MRC) a tutti gli stadi, molti centri nefrologici italiani hanno creato un team di nefrologi interventisti per gestire al meglio il paziente nella sua evoluzione clinica e per programmare un percorso personalizzato con la strategia più idonea per la terapia sostitutiva cronica.

Cercheremo di introdurre l'argomento rispondendo a due quesiti chiave, per provare a declinare i punti cardine di questa disciplina: la Nefrologia Interventistica

\section{Chi è il Nefrologo Interventista?}

È un medico specialista in nefrologia con un know-how ultraspecialistico dedicato all'argomento "accessi vascolari" ed

Received: January 4, 2020

Accepted: February 11, 2020

Published online: April 14, 2020

Indirizzo per la corrispondenza:

Carlo Jovane

UO Nefrologia e Dialisi ASST Valle Olona

PO "Sant'Antonio Abate" di Gallarate

Via Pastori, 4

21013 Gallarate, Varese - Italia

carlojovane@hotmail.it è parte di un team formato da altri specialisti e professionisti (2). In passato, alcuni specialisti in chirurgia generale e/o in urologia hanno seguito la vocazione per la nefrologia, mettendo al servizio le loro abilità chirurgiche; in tempi meno remoti molti nefrologi, specialmente nel nostro Paese, si sono dedicati "tout court" al confezionamento degli accessi vascolari o peritoneali nei loro pazienti dializzati; in tempi recenti, alcuni nefrologi hanno deciso di specializzarsi in chirurgia vascolare $o$ in radiologia, per poter perseguire il medesimo scopo; altri ancora hanno scelto un percorso personale, basato sull'acquisizione delle competenze necessarie, affidandosi alla saggia esperienza dei Nefrologi "anziani", con vivida passione ed esperienza nel campo. Ecco che, in questo scenario, è nata la figura del nefrologo interventista, con competenze chirurgiche, una grande passione e un ragionato timore per l'allestimento e la preparazione dell'accesso vascolare e/o dell'accesso peritoneale. La conditio sine qua non per tale scopo è una preparazione che potremmo definire "meta-nefrologica":

- competenze ultrasonografiche per la diagnostica vascolare e addominale, inserimento di CVC eco-guidati, biopsie renali con guida ecografica;

- conoscenza dettagliata dell'anatomia umana e topografica dei distretti anatomici di loro interesse e competenza;

- capacità di problem solving di accessi non funzionanti, siano essi CVC, FAV o cateteri peritoneali.

Dal momento che l'accesso vascolare è il mezzo di cui un paziente necessita per eseguire il miglior trattamento emodialitico possibile e che la qualità della terapia dialitica dipende principalmente dall'accesso, il principale compito di un nefrologo interventista è garantire tutto ciò. 


\section{Cos'è il Vascular Access Team?}

Dalla ricerca della perfezione e del miglioramento dei percorsi assistenziali, nasce il concetto del team multidisciplinare/ multiprofessionale: il Vascular Access Team, la cui attività ruota attorno all'accesso vascolare per emodialisi e che ha $\mathrm{i}$ seguenti ruoli principali:

- $\quad$ assicurare al paziente nefropatico un percorso assistenziale a 360 gradi, mediante il quale centrale la preservazione del patrimonio vascolare;

- $\quad$ Realizzare un accesso vascolare (AV) il più vicino possibile a quello ideale;

- massimizzare le percentuali di successo a breve e a lungo termine.

Il team è formato da uno o più nefrologi interventisti, da nefrologi clinici, da infermieri di dialisi dedicati agli accessi vascolari, da un chirurgo vascolare e/o da un radiologo interventista; il coordinatore del team dovrebbe essere il nefrologo interventista. In caso di malfunzionamento di una fistola, il nefrologo interventista mette in atto un lavoro di squadra per concordare l'iter diagnostico-terapeutico, dall'ecografia all'eco-colorDoppler, dalla TC dei distretti venosi centrali fino all'esame flebografico per valutare le necessità: procedere alla revisione chirurgica dell'accesso oppure eseguire eventuali angioplastiche della fistola. Alla stessa figura è generalmente devoluta l'organizzazione di sedute operatorie, naturalmente in collaborazione con gli altri specialisti e con personale non medico dedicato; ciò rappresenta lo scenario ideale per il raggiungimento degli obiettivi sia dei pazienti che degli operatori. Come noto, la stretta collaborazione in team di tutte queste figure può dare vita a percorsi assistenziali completi, efficaci e vincenti $(3,4)$.

Un tale livello di organizzazione ovviamente dovrà essere realizzato solo in strutture a grande complessità con disponibilità di modelli organizzativi innovativi. L'ormai noto modello Hub\&Spoke prevede l'esistenza di centri principali "di riferimento" (Hub) e di centri periferici (Spoke), dove l'attività chirurgica è minima o assente o dedicata solamente all'emergenza. Tali centri, afferendo come centri satelliti (Spoke), collaborano con il polo nefrologico principale (Hub), dove è presente il Vascular Access Team, che accoglie tutti i pazienti con complicanze degli accessi vascolari. II team degli accessi vascolari ubicato presso la Nefrologia di un centro Hub è il riferimento di tutto il bacino di utenza del territorio, dove si trovano i "suoi" centri Spoke, i quali dovranno garantire un orientamento diagnostico quantomeno iniziale sulla problematica intercorsa.

Alla luce di quanto soprascritto, cercheremo di descrivere due realtà nefrologiche regionali distanti e diverse, analizzandone pregi e difetti.

\section{Esperienza dell'ASST Valle-Olona di Varese}

Per effetto della legge regionale $n^{\circ} 23$ dell'11 agosto 2015, il sistema sanitario della Lombardia ha subito una radicale riforma con la nascita delle Agenzie di Tutela della Salute (ATS) e delle Aziende Socio-Sanitarie Territoriali (ASST). Le Agenzie di Tutela della Salute (ATS), in sostituzione delle precedenti Aziende Sanitarie Locali (ASL), sono distribuite su tutto il territorio della Lombardia. Hanno compiti di gestione, programmazione e controllo; sono 8: Insubria (Varese e Como), Brianza (Monza e Lecco), Bergamo, Brescia, Pavia, Val Padana (Cremona e Mantova), Città Metropolitana (Milano e Lodi) e quella della Montagna (Sondrio, Valtellina e Valcamonica). Ogni ATS è suddivisa in diversi dipartimenti specializzati nelle singole funzioni e incorpora le ASST, che hanno sostituito formalmente le precedenti Aziende Ospedaliere (AO). Le ASST sono 27 e spetta loro l'onere di erogare le prestazioni socio-sanitarie. Dal portale della Regione Lombardia: “... Le Aziende Socio-Sanitarie Territoriali (ASST) concorrono all'erogazione dei livelli essenziali di assistenza (LEA) e di eventuali livelli aggiuntivi definiti dalla Regione... Le ASST si articolano in due settori aziendali, rispettivamente definiti rete territoriale e polo ospedaliero. II settore aziendale polo ospedaliero si articola in presidi ospedalieri e/o in dipartimenti organizzati in diversi livelli di intensità di cura, in coerenza con il regolamento sugli standard ospedalieri. Questo settore è prevalentemente dedicato al trattamento del paziente in fase acuta ed è sede dell'offerta sanitaria specialistica. Al settore aziendale rete territoriale delle ASST afferiscono i presidi ospedalieri territoriali (POT) che sono strutture multiservizio deputate all'erogazione di prestazioni residenziali sanitarie e sociosanitarie a media e a bassa intensità per acuti e cronici e di prestazioni ambulatoriali e domiciliari (5)".

L'azienda Ospedaliera "S. Antonio Abate" di Gallarate è stata unita con altri ospedali, creando l'ASST Valle Olona, accentrando i servizi di singole Aziende Ospedaliere in un unico centro, che racchiude le realtà di 4 ospedali (Gallarate, Busto Arsizio, Saronno, Somma Lombardo).

L'ASST Valle Olona ha un bacino di utenza di circa 450,000 abitanti, che riguarda principalmente la zona SUD della provincia di Varese, in contrapposizione con I'ASST-Settelaghi della parte Nord. Il servizio di dialisi ambulatoriale è articolato su due centri di dialisi ospedaliera (Gallarate e Busto Arsizio) e su tre Centri di Assistenza Limitata (CAL), vale a dire con una presenza "solo" settimanale (limitata) del medico. I pazienti dei CAL, selezionati secondo parametri clinici, sono pari a 84 (24 a Somma Lombardo e Castellanza, 36 a Saronno). Nei centri ospedalieri vengono dializzati cronicamente circa 220 pazienti; in aggiunta, vengono eseguite almeno 50 dialisi/ anno per i pazienti incidenti acuti, con un range di 350-400 pazienti in dialisi/anno.

Il team degli accessi vascolari per dialisi è formato da due nefrologi interventisti (uno per centro ospedaliero), due chirurghi vascolari, dedicati agli accessi (scelti in collaborazione 
con il Direttore della Chirurgia Vascolare) e tre infermieri, che collaborano in sala operatoria per l'allestimento. II radiologo interventista non è presente, giacché l'attività interventistica endovascolare è eseguita routinariamente dal chirurgo vascolare. L'ambulatorio dei pazienti uremici, equi-distribuito tra Busto Arsizio e Gallarate, gestito da un nefrologo clinico e da un infermiere, è dedicato a tutti coloro che hanno una MRC dal IV stadio in avanti (GFR $<30 \mathrm{~mL} / \mathrm{min}$ ); per tutti i pazienti "progressor" verso il V stadio, il nefrologo propone al paziente il percorso della predialisi. Secondo procedure aziendali codificate, il Programma Educativo di predialisi è articolato in 4 incontri, di circa 30 minuti, con un referente educativo infermiere (sono 6 infermieri a rotazione), diretti dal Coordinatore Infermieristico.

Gli incontri sono cosi articolati:

- ascolto e conoscenza della persona e del suo contesto familiare: alleanza terapeutica;

- informazioni su funzione renale, malattia e dialisi e tipi di dialisi (extracorporea o peritoneale);

- come vivere la terapia dialitica, cosa mangiare, come vestirsi e cosa fare;

- terapia dietetica del dializzato, con scheda di compilazione comparativa al tempo zero e dopo 6 mesi.

È questo è il momento in cui inizia la collaborazione con il nefrologo interventista, con il quale si analizza nel dettaglio I'anamnesi e si discute sull'allestimento dell'accesso dialitico. L'equipe medico/infermieristica decide insieme al paziente e ai familiari/caregiver la metodica di sostituzione della funzione reale persa (extracorporea e/o peritoneale), discutendo sulle eventuali indicazioni/controindicazioni e analizzandole.

Per la dialisi peritoneale, viene compilato un questionario che analizza le criticità cliniche, psico-socio-attitudinali e logistiche, specialmente igienico-abitative. Se la valutazione complessiva dà un esito positivo, il nefrologo interventista insieme al chirurgo generale prepara il paziente per una valutazione clinica e la pianificazione dell'intervento di posizionamento.

In casi selezionati, quando l'esame obiettivo mostra la presenza di ernie ombelicali e inguinali o di altre ernie della parete addominale, è indicata l'esecuzione di un'ecografia della parete addominale. Se l'esame non è dirimente, è utile l'esecuzione di una TC della parete addominale senza mezzo di contrasto. In caso positivo (presenza di ernie), viene eseguito l'intervento di ernioplastica, propedeutico al posizionamento del catetere peritoneale. Nel caso di pregressi interventi chirurgici della parete addominale, con possibile presenza di aderenze dell'omento, in cavità peritoneale, si procederà ad una contestuale laparoscopia esplorativa; se la stessa dovesse dare esito negativo, si procederà al posizionamento del catetere peritoneale con tecnica chirurgica "open"; se, invece, l'esame laparoscopico mostrasse delle aderenze estese, si procederà al posizionamento con guida laparoscopica in cavità addominale con eventuali interventi di lisi aderenziali, qualora fosse necessario. In base alla tempistica dettata dal nefrologo, viene programmato il posizionamento del catetere peritoneale, in sala operatoria, da parte del chirurgo e del nefrologo.

Per la dialisi extracorporea, il nefrologo interventista pone indicazione sul tipo di $\mathrm{AV}$ a cui il paziente risulta idoneo.

Se i pazienti sono idonei all'allestimento di una FAV, il nefrologo interventista mette in atto il percorso clinico per il confezionamento; effettua un mapping clinico con esame obiettivo delle braccia per verificare la presenza di cicatrici, tatuaggi, flogosi cutanea, edemi e circoli collaterali del cingolo scapolare, asimmetria degli arti, trofismo ed elasticità della cute e valutazione del polsi arteriosi periferici. Esegue un mapping ecografico completo del braccio dal cingolo scapolare fino ai polsi con studio delle vene e delle arterie, corredato da test di ischemia e dal test ecografico di Allen, con studio dell'arcata palmare $(6,7,11)$.

L'esame clinico viene messo in correlazione con le eventuali comorbidità presenti (diabete, vasculopatia, patologie trombofiliche, pregressi posizionamenti di CVC in vene giugulari/succlavie, terapia in corso con steroidi e anticoagulanti) (7), il tutto per decidere l'iter più appropriato ed eventuali esami aggiuntivi. Il dato della presenza di calcificazioni arteriose è spesso prognosticamente sfavorevole per la presenza di un flusso arterioso (inflow) insufficiente a garantire una portata adeguata alla FAV e può richiedere una revisione critica del caso.

La flebografia pre-allestimento con mdc (per motivi organizzativi non si usa la flebografia con $\mathrm{CO} 2$ ) viene eseguita se l'esame obiettivo mostra segni di stenosi venosa centrale (cfr. supra), nei pazienti con pregressi posizionamenti di CVC e, infine, anche nell'evidenza ecografica di un sistema venoso superficiale insufficiente (pazienti diabetici, affetti da ADPKD), con rischio di early failure da insufficiente flusso venoso (outflow). L'uso del mdc, comunque, viene ristretto solo a casi selezionati di pazienti per i quali l'ingresso in dialisi è imminente (4-6 mesi).

La FAV nativa viene allestita all'arto superiore non dominante, in direzione distale-prossimale, nell'ottica di risparmio e conservazione del patrimonio venoso del paziente $(7,11)$ :

- FAV distale al polso (1/3 distale di avambraccio) con anastomosi "side-to-side" (STS), tra arteria radiale e vena cefalica, con legatura distale del ramo venoso afferente alla mano; I'anastomosi "end-to-side" rimane una valida alternativa alla prima ed è stata oggetto di numerose evidenze scientifiche, allo scopo di individuare le cause più frequenti dell'"early-failure" (8);

- in caso di fallimento della fistola radio-cefalica al polso, si procede con una FAV prossimalizzata middle arm (9), 
al terzo prossimale di avambraccio, con anastomosi "side-to-side" (STS) tra arteria radiale all'origine e vena mediana-cefalica. Questa scelta spesso viene utilizzata come "prima scelta" nei pazienti diabetici, con arteriopatia periferica a carico dell'arto superiore;

- $\quad F A V$ prossimale, secondo Gracz (omeralo-cubitale) al terzo prossimale di avambraccio (10);

- $\quad$ FAV prossimale, al terzo distale di braccio, con anastomosi "side-to-side" (STS) brachio-cefalica o brachiomediana.

I due nefrologi interventisti eseguono di routine questo tipo di FAV, dette anche FAV di I livello, vale a dire un intervento di anastomosi senza ulteriori complicanze o necessità di altri tipi di interventi vascolari;

- FAV prossimale omero-basilica, che è la FAV che più frequentemente necessita di trasposizione/superficializzazione di vena. Viene presa in considerazione dopo trombosi o malfunzionamento di una FAV prossimale, ma anche come prima istanza nei pazienti con pressioni arteriose basse o a rischio di infezioni. Questo tipo di FAV viene indicato, anche, con il termine di FAV di II livello, per indicare la necessità di un intervento vascolare ulteriore sui vasi, oltre al confezionamento dell'anastomosi. Queste FAV vengono eseguite in sala operatoria dal chirurgo vascolare, dopo una discussione collegiale e una pianificazione dell'intervento, insieme al nefrologo interventista.

Se il mapping ecografico esclude una FAV nativa, in valutazione collegiale, si propone l'allestimento di una FAV protesica (retta, loop) e, anche in questo caso, il nefrologo interventista partecipa come operatore.

Se le condizioni cliniche controindicano le altre scelte, viene organizzato il posizionamento di CVC long term. Previo studio ecografico delle logge laterali del collo, dello stato coagulativo e della terapia in corso, il nefrologo interventista, in autonomia, procede, con il supporto del personale infermieristico, al posizionamento in sala emodinamica, con amplificatore di brillanza, del CVC, con puntura venosa ecoguidata. Per ogni paziente viene scelto il tipo di CVC:

- Lunghezze diverse in base alla statura del paziente e/o al sito di inserzione (VGI destra o sinistra), che saranno rispettivamente di 190-240-270 $\mathrm{mm}$.

- Geometria del tip (punta del CVC) in base alla corporatura; Tip splittato, vale a dire con le punte arteriosa e venosa separate e lontane tra loro come fossero due CVC indipendenti; Tip palindromo, (rá $\lambda \iota v$ "di nuovo" e $\delta \rho o ́ \mu o \varsigma$ "percorso", "percorso in entrambi i sensi"), sono due punte identiche, l'una lo specchio dell'altra.

- II CVC retrogrado, è un CVC doppio lume, di solito non precurvato, che va inserito in Vena e successivamente viene tunnellizzato nel sottocute e infine montato col apposite prolunghe che saranno rispettivamente il lume arterioso e il lume venoso; il CVC anterogrado è un CVC completo, di solito precurvato, che prevede prima la tunnellizzazione anteriore nella loggia pettorale sottocutanea (da qui il nome ante-rogrado) e solo successivamente l'inserimento in Vena, su sistema peel way.

II follow-up delle FAV native e protesiche prevede due livelli di sorveglianza; il primo viene eseguito dal gruppo infermieristico della sorveglianza degli accessi, che monitora con parametri clinici e dialitici la "salute" delle FAV. Su loro indicazione, si allerta il nefrologo interventista per la sorveglianza di secondo livello con ecografia ed ECD. Il nefrologo interventista attua un piano strategico con il chirurgo e predispone un eventuale intervento di revisione per ogni singolo caso.

Nonostante il numero limitato di nefrologi (11 di cui 5 a Gallarate e 6 a Busto Arsizio) che si occupano di altre attività cliniche, grazie anche alla disponibilità dei chirurghi, annualmente viene eseguito un numero consistente di interventi:

- $\quad 130$ CVC long term tunnellizzati (nefrologo interventista, in autonomia);

- 60 CVC short term per dialisi urgenti (nefrologo interventista, in autonomia);

- 50 FAV native di avambraccio distali/prossimalizzate/ prossimali (2 nefrologi interventisti);

- 20 FAV di braccio prossimali, brachio-basiliche, con necessità di trasposizione/superficializzazione di vena (chirurgo vascolare e nefrologo interventista);

- 20 FAV protesiche (chirurgo vascolare e nefrologo interventista);

- 40 interventi di accessi vascolari compromessi (riduzione di aneurismi, "bridge" protesici con salvataggio della FAV) (chirurgo vascolare + nefrologo interventista);

- 30 posizionamenti di cateteri peritoneali (chirurgo generale e nefrologo interventista);

- 40 biopsie renali eco-guidate (nefrologo interventista).

\section{Esperienza dell’Ospedale Sant'Eugenio, ASL Roma 2, Roma}

Nell'organizzazione delle ASL, la Regione Lazio si avvicina meglio al modello-sistema Hub\&Spoke. Dispone, infatti, di diversi centri di riferimento per l'accesso vascolare (Hub), di cui uno dei più grandi e con maggiore attività inerente all'accesso dialitico è l'Ospedale Sant'Eugenio, collocato all'interno dell'ASL Roma 2. L'ASL Roma 2, una delle più grandi d'Italia, si estende per circa $470 \mathrm{~km}^{2}$ nella zona sud-est del Comune di Roma e conta oltre $1.300,000$ abitanti (il $45 \%$ della popolazione complessiva residente di tutto il Comune). È suddivisa in 6 distretti sanitari che coincidono con i municipi (dal IV al IX). Dal Rapporto annuale RRDTL (Registro Regionale Dialisi e Trapianto Lazio) si evince che il numero 
dei pazienti emodializzati cronici prevalenti (>18 anni) nella Regione Lazio è di circa 4.700 e dei pazienti in dialisi peritoneale è di circa 400.

L'ASL Roma 2 comprende 15 centri dialisi tra pubblico e privato, di cui un centro in Azienda Universitaria che è il Policlinico di Tor Vergata. I pazienti in emodialisi in carico al Sant'Eugenio sono 130 distribuiti tra l'ospedale e le 2 unità decentrate di dialisi (Santa Caterina della Rosa e CTO Alesini).

Il paziente in predialisi (GFR $<30 \mathrm{~mL} / \mathrm{min}$ ) è seguito presso un ambulatorio di II livello. In fase di programmazione relativa alla metodica dialitica e all'allestimento dell'accesso, il paziente viene seguito presso l'ambulatorio educazionale, dove viene accompagnato nella scelta del trattamento sostitutivo sulla base delle necessità cliniche e delle condizioni psicologiche e sociali attraverso un percorso e dei protocolli prestabiliti.

Per quanto riguarda l'accesso vascolare, la valutazione obiettiva e strumentale (prime FAV, controlli degli accessi vascolari in pazienti già dializzati) con il conseguente monitoraggio avviene presso l'ambulatorio degli accessi vascolari articolato in due giorni a settimana, il martedì e il venerdi, per garantire a tutti i pazienti di potersi recare al controllo in un giorno interdialitico. II monitoraggio dell'accesso vascolare avviene per il primo anno ogni tre mesi e, per gli anni successivi, ogni 6 mesi, salvo diverse indicazioni o necessità cliniche.

Il numero di accessi dell'ambulatorio degli accessi vascolari è di circa 1200 pazienti/anno. In tale occasione viene eseguito il monitoraggio ecografico ed eco-color-Doppler dell'accesso vascolare con l'indicazione di eventuali esami strumentali di secondo livello (angio-TC del distretto venoso centrale, flebografia con mdc organo-iodato o con $\mathrm{CO} 2$ e angio-TC del distretto arterioso periferico con ricostruzione 3D). L'angio-TC del distretto venoso centrale è indicata quando si sospettano stenosi del distretto venoso centrale (pazienti con precedenti cateterismi venosi centrali, edema del braccio della FAV, edema del volto ed edema della mano). L'esame flebografico viene eseguito quando esiste il sospetto di stenosi della vena di outflow, senza evidenza ecografica di un aumento delle resistenze, o qualora l'esame ecografico non sia soddisfacente. L'angio-TC con ricostruzione 3D trova indicazione nel paziente arteriopatico con "sindrome da furto", con dolore ischemico o con parestesie dell'arto o nel paziente con evidenti calcificazioni vascolari, quando l'esame ecografico non risulta dirimente.

L'attività chirurgica si articola su tre sedute operatorie settimanali dedicate al paziente nefropatico. Le sedute operatorie vengono stabilite la settimana precedente per la successiva seguendo l'ordine di urgenza e di inserimento in lista d'attesa. In genere, tale intervallo di tempo non supera i 30 giorni, salvo in situazioni d'urgenza non differibili, in pazienti in terapia conservativa, per i quali è necessario tenere conto del tempo di maturazione dell'accesso prima dell'inizio della dialisi, o nel paziente candidato alla dialisi peritoneale che necessita del tempo di stabilizzazione e addestramento alla dialisi. Due volte a settimana, in giorni prestabiliti, nefrologi interventisti e nefrologi clinici si riuniscono per discutere casi clinici rilevanti (medici o chirurgici) e per pianificare le sedute operatorie successive.

II Sant'Eugenio di Roma offre un servizio di reperibilità "h24" ai pazienti emodializzati sia cronici che acuti; eroga prestazioni per molti centri di dialisi ospedaliera della Regione (almeno 4) e per centri dialisi del territorio dell'ASL e fuori ASL (in numero di 9). La stima dei pazienti seguiti per l'accesso vascolare è di circa 700 . Vengono trattati circa 400 pazienti acuti/anno.

II team degli accessi vascolari del Sant'Eugenio è formato da 4 nefrologi interventisti, di cui 1 specialista anche in chirurgia vascolare (il primario, coordinatore del team), 1 chirurgo vascolare e 2 radiologi interventisti. II personale non medico dedicato è formato da 1 coordinatore infermieristico, 4 infermieri di sala, 2 strumentisti e 2 ausiliari. I medici nefrologi clinici coadiuvano l'attività chirurgica nella gestione del paziente nel reparto di degenza e sono coordinati da un Responsabile. II Data Manager raccoglie ed elabora i dati relativi all'attività clinica, chirurgica e di dialisi del reparto.

La scelta dei vasi da utilizzare per l'accesso vascolare è sempre dettata dalle caratteristiche del paziente e segue la logica della preservazione del patrimonio vascolare. La tecnica chirurgica è quella microvascolare, con l'allestimento di anastomosi latero-terminali anche su vasi di dimensioni $<2,5 \mathrm{~mm}$. L'accesso chirurgico per le FAV distali, prossimalizzate o prossimali in genere non supera i $3 \mathrm{~cm}$ di lunghezza. L'anastomosi è sempre $\leq 4 \mathrm{~mm}$ per impedire fenomeni di iperafflusso. II centro è stato inoltre tra i primi in Italia a impiantare il dispositivo VasQ su vasi nativi, supporto in nitinolo esterno all'anastomosi che impedisce l'inginocchiamento del vaso, consente una corretta e stabile angolazione tra arteria e vena, ma, soprattutto, riduce il fenomeno dell'iperafflusso "contenendo" la regione perianastomotica e riducendo l'iperplasia dell'intima (6). È il centro in Italia con la maggiore casistica di impianto.

Le FAV protesiche vengono impiantate con disponibilità di diversi modelli in termini di configurazione (loop, retta), di materiale (Omniflow, Gore) e di rapidità di utilizzo.

I CVC tunnellizzati vengono posizionati in sala operatoria con l'ausilio dell'amplificatore di brillanza e, in molti casi, presso la sala angiografica della radiologia interventistica. Il primo operatore è sempre il nefrologo interventista. I dispositivi a disposizione sono tutti a punta simmetrica, sia anterogradi che retrogradi. In casi selezionati vengono posizionati cateteri di Tesio. Il supporto del chirurgo vascolare è richiesto in caso di riduzione di grandi aneurismi o di interventi di maggiore complessità chirurgica. II supporto del radiologo interventista è richiesto in caso di diagnostica strumentale di secondo livello e in caso di necessità di correzione di stenosi centrali, trombosi di protesi o FAV native e stenosi periferiche. La tromboaspirazione della FAV trova indicazione in tutte le trombosi recenti ( $<48$ ore), anche nelle trombosi di FAV distali, prima di procedere all'approccio chirurgico. La percentuale di successo è del $98 \%$. La trombolisi 
con urokinasi o altri agenti trombolitici è sempre l'approccio di seconda scelta.

Il Vascular Access Team del Sant'Eugenio esegue:

- 400 interventi/anno di chirurgia dell'accesso vascolare:

- 200 FAV native avambraccio (distali, prossimalizzate)

- 125 FAV native braccio (omero-cefalica, omerobasilica con trasposizione e superficializzazione)

- 35 FAV protesiche

- 40 interventi di accessi vascolari complessi (riduzione di aneurismi, "bridge" protesici con salvataggio della FAV);

- 60 interventi di posizionamento di cateteri peritoneali;

- 300 posizionamenti di CVC tunnellizzati;

- 70 procedure di radiologia endovascolare per accessi vascolari complicati o stenosi centrali;

- 70 biopsie renali eco-guidate.

\section{Conclusioni}

Un numero ancora troppo esiguo di centri di nefrologia italiani è dotato di un team dedicato agli accessi vascolari.

Presso I'ASST Valle Olona, il programma nefrologico di un ambulatorio di predialisi permette una valutazione complessiva del paziente. Il team degli accessi vascolari di dialisi non è concepito per un unico "centro".

Le ASST lombarde sono state organizzate in modo che ognuna di esse sia il riferimento per un determinato bacino d'utenza e affinché ognuna abbia le risorse umane ed economiche per offrire prestazioni di altissimo livello per gli ospedali che non hanno una nefrologia con un livello di organizzazione strutturato. Ogni macroarea è dotata di una propria autonomia di gestione globale dell'accesso dialitico e delle procedure nefrologiche invasive.

Nel Lazio, l'organizzazione è concepita in modo differente: i reparti di nefrologia laziali sono concepiti sul modello dell'Hub\&Spoke, secondo cui il costo di un Vascular Access Team sarebbe raramente giustificato in un reparto di nefrologia, anche se fosse di grandi dimensioni. La maggior parte delle dialisi del territorio non può permettersi un'organizzazione strutturale di questo tipo, non disponendo, spesso, di una sala operatoria e/o di nefrologi interventisti. I centri Hub sono concepiti per rendere qualitativamente e quantitativamente valido il lavoro di un Vascular Access Team e anche per dare una dignità scientifica al lavoro chirurgico. In termini qualitativi, questo sistema sarà indispensabile sia per il nefrologo clinico, che potrà fare riferimento al team degli accessi vascolari per certificare le caratteristiche chirurgiche della fistola arterovenosa da utilizzare, sia per il paziente, che richiede, sempre di più, un'elevata qualità delle prestazioni chirurgiche.

Verosimilmente, la medicina legale, in futuro, potrebbe non giustificare l'operato di un singolo nefrologo che effettui pochi interventi o che lavori in un centro "piccolo".
In termini quantitativi, bisogna considerare l'industrializzazione di un processo multidisciplinare/professionale che comprende sia la diagnostica che la chirurgia, garantendo una qualità massima a qualsiasi paziente. I "grandi" numeri con cui un team multidisciplinare/professionale può validare la bontà delle scelte chirurgiche creano delle basi scientifiche, sulle quali poter proporre idee innovative, basate su esperienza ed evidenza.

Entrambi i sistemi hanno dei punti di forza e spunti di riflessione.

II sistema Hub\&Spoke pone le basi sul concetto dei grandi numeri e di un team dedicato quasi esclusivamente a un'intensa attività chirurgica, che deve rendere al massimo con un'efficienza al limite della perfezione, su cui gli ospedali "piccoli" devono poter contare per la risoluzione dei problemi nel più breve tempo possibile.

Il sistema lombardo non è cosi estremo nelle scelte, ma, concettualmente, segue lo stesso percorso: più team multidisciplinari/professionali, che lavorano in modo efficiente sulle medesime problematiche. Non esistono nefrologie piccole e periferiche senza mezzi economici; esistono dialisi periferiche senza la costante presenza di un medico (CAL) che necessitano di una nefrologia "grande" che possa assistere i propri pazienti in tempi ragionevoli, con un'efficienza e una professionalità ai massimi livelli.

\section{Disclosures}

Financial support: The authors declare no financial support for this manuscript.

Conflict of interest: The authors declare no conflict of interest.

\section{Bibliografia}

1. Efstratiadis G, Platsas I, Koukoudis P, Vergoulas G. Interventional Nephrology: a new subspecialty of Nephrology. Hippokratia. 2007;11(1):22-4.

2. Jovane C. Gli accessi vascolari: il punto di vista di un giovane nefrologo. Giornale di Tecniche Nefrologiche \& Dialitiche. 2013;25(4):352-3.

3. Salman L, Beathard G. Interventional nephrology: Physical examination as a tool for surveillance for the hemodialysis arteriovenous access. Clin J Am Soc Nephrol. 2013;8(7): 1220-7.

4. Chan MR. Interventional nephrology: What the nephrologist needs to know about vascular access. Clin J Am Soc Nephrol. 2013;8(7):1211-2.

5. https://www.regione.lombardia.it/wps/portal/istituzionale/ HP/DettaglioServizio/servizi-e-informazioni/Cittadini/salute-eprevenzione/strutture-sanitarie-e-sociosanitarie/ser-aziendeospedaliere-sal/aziende-socio-sanitarie-territoriali, Accessed March 12, 2020.

6. Napoli M. Eco Color Doppler \& Accessi Vascolari per Emodialisi. Wichtig Editore. 2010. ISBN: 8873210317.

7. Segoloni G, Leonardi G (collaboratore). Gestione fistola arterovenosa per emodialisi. www.nephromeet.com/web/eventi/ NEPHROMEET/index.cfm. 15 maggio 2013, Accessed March 12, 2020. 
8. Bharat A, Jaenicke M, Shenoy S. A novel technique of vascular anastomosis to prevent juxta-anastomotic stenosis following arteriovenous fistula creation. J Vasc Surg. 2012;55(1): 274-80.

9. Bonforte G, Zerbi S, Surian M. The middle-arm fistula: A new native arteriovenous vascular access for hemodialysis patients. Ann Vasc Surg. 2004;18:448-52.
10. Gracz KC, Ing TS, Soung LS, Armbruster KF, Seim SK, Merkel FK. Proximal forearm fistula for maintenance hemodialysis. Kidney Int. 1997;11:71-5.

11. Gessaroli M (Coordinatore), Alessi Innocenti A, Carbonari L, De Donato G, Dorigo W, lob G, Morelli I, Parente B. Linee Guida Sicve 2015. Accessi Vascolari. The Italian Journal of Vascular and Endovascular Surgery 2015.September;22(3 Suppl 2):95-116. 